Revue de droit comparé du travail et de la sécurité sociale

3 | 2020

La Directive 2019/1158 du 20 juin 2019 concernant l'équilibre entre vie personnelle et vie privée des parents et des aidants

Mise en œuvre en Pologne de la Directive (UE) 2019/1158 du Parlement européen et du Conseil du 20 juin 2019 concernant l'équilibre entre la vie professionnelle et la vie privée des parents et des aidants

Anna Musiała

\title{
OpenEdition
}

Édition électronique

URL : https://journals.openedition.org/rdctss/971

DOI : $10.4000 /$ rdctss. 971

ISSN : 2262-9815

Éditeur

Centre de droit comparé du travail et de la sécurité sociale

Édition imprimée

Date de publication : 1 novembre 2020

Pagination : 94-99

ISSN : 2117-4350

Référence électronique

Anna Musiała, « Mise en œuvre en Pologne de la Directive (UE) 2019/1158 du Parlement européen et du Conseil du 20 juin 2019 concernant l'équilibre entre la vie professionnelle et la vie privée des parents et des aidants ", Revue de droit comparé du travail et de la sécurité sociale [En ligne], 3 | 2020, mis en ligne le 01 novembre 2021, consulté le 11 novembre 2021. URL : http:// journals.openedition.org/rdctss/971; DOI : https://doi.org/10.4000/rdctss.971

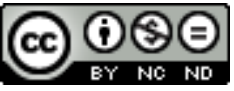

Revue de droit comparé du travail et de la sécurité sociale est mise à disposition selon les termes de la Licence Creative Commons Attribution - Pas d'Utilisation Commerciale - Pas de Modification 4.0 International. 


\section{Mise en cuvre en Pologne de LA DIRECTIVE (UE) 2019/1158 DU PARLEMENT EUROPÉEN ET DU CONSEIL DU 20 JUIN 2019 CONCERNANT L'ÉQUILIBRE ENTRE LA VIE PROFESSIONNELLE ET LA VIE PRIVÉE DES PARENTS ET DES AIDANTS}

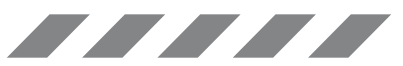

\section{ABSTRACT}

The aim of this study is to analyse the possible effects of the Polish legislator's implementation of the directive of the European Parliament and the Council (EU) 2019/1158 of 20 June 2019 concerning the work-life balance of parents and aid, repeal of the 2010/18 /EU Council Directive.

KEYWORDS: Work-life Balance, EU Directive 2019/1158, Poland.

\section{RÉSUMÉ}

La présente étude a pour objet d'analyser les effets possibles de la mise en œuvre par le législateur polonais de la directive du Parlement européen et du Conseil (UE) 2019/1158 du 20 juin 2019 concernant l'équilibre entre la vie professionnelle et la vie privée des parents et des aidants, abrogeant la directive 2010/18 / UE du Conseil.

MOTS CLÉS: Équilibre vie professionnelle et privée, directive UE 2019/1158, Pologne. 


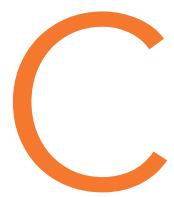

onformément aux dispositions des considérants de la directive, il s'agit d'améliorer le fonctionnement des États dans le domaine de l'égalité des chances sur le marché du travail, et du traitement des femmes et des hommes au travail en raison de leurs responsabilités familiales et d'aidants. Cette directive répond aux besoins liés aux changements démographiques qui affectent aussi la Pologne, à la nécessité d'approfondir le fonctionnement du principe de l'égalité des sexes résultant des actes juridiques adoptés pour la Communauté, aux besoins liés à l'activation professionnelle des femmes et à la lutte contre l'exclusion en raison de soins donnés aux mineurs.

Le soin donné à la normalisation de ces questions revêt une importance particulière dans le contexte polonais dans la mesure où, selon les données d'Eurostat, la part des femmes dans la tranche d'âge 15-64 ans travaillant ou cherchant activement du travail était de $63 \%$ en 2018, soit en cinquième position dans cette étude statistique ${ }^{1}$. Cependant, il convient de noter que l'âge de la retraite des femmes en Pologne est en principe de 60 ans.

Par ailleurs, environ $75 \%$ des femmes sans emploi et ne recherchant pas d'emploi motivent leur choix de rester à la maison par le fait de s'occuper de leurs enfants et d'autres responsabilités familiales ${ }^{2}$. Cette situation résulte directement du nombre insuffisant de places dans les crèches et les écoles maternelles. Enfin, cette situation est d'autant plus renforcée que $40 \%$ des salariées ne peuvent pas bénéficier d'horaires de travail souples et que $77 \%$ des Polonais considèrent que le fait de prendre soin de la maison et de la famille relève des fonctions les plus importantes d'une femme ${ }^{3}$.

L'art. 4 de cette directive oblige les États membres à prendre les mesures nécessaires pour que les pères ou les seconds parents aient le droit de prendre un congé de paternité de dix jours ouvrables, à l'occasion de la naissance de l'enfant du travailleur, indépendamment de la période d'ancienneté et indépendamment de leur situation civile ou familiale. Étant donné qu'en Pologne il n'existe pas de réglementation concernant les partenariats (PACS) et qu'en conséquence ils sont omis de la réglementation relative aux relations parentales, l'obligation de mise en œuvre ne s'appliquera pas aux couples de même sexe. Dans les six premiers mois de 2019, 88500 hommes ont eu recours au congé de paternité4.

Dans la loi polonaise, le congé de paternité est géré par l'article $182^{3}$ du Code du travail. Il a été séparé du dénommé « congé de papa » avec lequel il est parfois confondu et qui constitue un moyen de transférer le congé de maternité à un père. Le paragraphe 1 donne au père élevant un enfant (il ne doit pas être l'époux de la mère de l'enfant) le droit à un congé de paternité de 2 semaines maximum, strictement limité aux 24 mois de l'enfant, ou aux 24 mois écoulés depuis la décision définitive statuant l'adoption de l'enfant (l'enfant

1 A. Rozwadowska, «Polska to nie jest kraj dla matek. Dlaczego tak dużo kobiet nie pracuje? » [La Pologne n'est pas un pays pour les mères. Pourquoi autant de femmes ne travaillent pas?] : https:// wyborcza.pl/7,155287, 25604754,polska-to-nie-jest-kraj-dla-matek.html (2020).

2 Ibid.

3 lbid.

4 https://www.gov.pl/web/rodzina/rodzice-na-urlopie2 
doit être âgé de moins de 7 ans) ou reportant la scolarité obligatoire de l'enfant (son âge maximal est alors de 10 ans).

Par ailleurs, le droit à un congé de paternité ne peut théoriquement être exercé qu'une seule fois, deux exceptionnellement, dont chacune ne peut durer moins d'une semaine. Ainsi, le Code du travail offre aux travailleurs-pères une solution plus avantageuse que celle adoptée dans la directive. Seul le travailleur décide du moment où il exercera le droit à ce congé, son unique obligation étant de respecter la durée statuée par le législateur. Le travailleur-père doit communiquer sa volonté d'exercer son droit au congé, en respectant un préavis de 7 jours, et effectue une demande en ce sens auprès de son employeur. En pratique, le congé de paternité peut être accordé exclusivement à un père élevant un enfant mais refusé par exemple à ceux qui remplissent le rôle d'aidant assurant l'éducation $d^{\prime} u n$ enfant ${ }^{5}$. L'interprétation de l'article 4 semble indiquer le besoin du " deuxième parent » de recourir plus largement à ce type de congé, car c'est le seul moyen de réaliser l'objectif indiqué dans le préambule.

L'article 5 de la directive, relatif au congé parental, exige de prendre les mesures nécessaires pour que chaque travailleur ait le droit individuel de prendre un congé parental de quatre mois avant que l'enfant n'atteigne un âge déterminé, dont deux mois intransférable à un deuxième parent. Pour ce type de congé, le Code du travail polonais s'appuie sur des dispositions basées sur d'autres critères. Tout d'abord, le législateur polonais octroie aux deux parents 32 ou 34 semaines de congé, qu'ils peuvent diviser à leur discrétion. Néanmoins, aucune partie du congé parental ne peut être d'une durée inférieure à 8 semaines (sauf rares exceptions). Dans la pratique, ceci signifie que le droit au congé peut être exercé par exemple uniquement par la mère, ou uniquement par le père, ou par tous les deux en même temps (le cas échéant, ils n'exerceront leur droit au congé que 16 semaines). Une autre solution consiste également dans le fait que le droit au congé n'est exercé que par un seul parent, le deuxième recevant une allocation pour une période correspondant à ce congé.

La directive offre ainsi la possibilité de diviser le congé parental en 16 semaines pour chacun des deux parents, étant entendu que seule la moitié de ce congé pourra être transférée à un deuxième parent.

Actuellement, le droit au congé parental ne peut être exercé que jusqu'à la fin de l'année civile au cours de laquelle l'enfant atteint l'âge de 6 ans. Par ailleurs, le congé parental n'est pas accordé en fonction de critères tels que l'ancienneté ou les besoins spécifiques de l'employeur. Ce dernier doit prendre en compte la demande de congé dans la mesure où il est considéré comme un élément particulier de protection des femmes salariées.

En Pologne, la situation juridique actuelle est également conforme à l'article 5, al. 6 de la directive. Ainsi, en vertu de l'article $182^{1 \mathrm{e}} \S 1$ du Code du travail, « le travailleur peut combiner l'exercice de son droit au congé parental avec le travail exécuté chez l'employeur qui lui avait accordé ce congé, mais le temps de travail ne peut être supérieur à la moitié du temps plein. Le cas échéant, le congé parental est accordé pour la partie restant du plein temps ». Cette possibilité offerte au parent de bénéficier d'une allocation lorsque le deuxième parent exerce son droit au congé semble avantageuse.

5 E. Maniewska, «Art. 182(3)», Komentarz aktualizowany do Kodeksu pracy [Commentaire au Code du travail], LEX/el., 2020. 
La directive entraîne par ailleurs le prolongement (par rapport à la législation actuellement en vigueur en Pologne) du congé d'aidant jusqu'à 5 jours ouvrables dans l'année. La solution adoptée en Pologne permettant d'octroyer le congé en heures - signifiant que le congé d'un travailleur à temps partiel est accordé au prorata de son temps de travail - ne paraît pas contraire à la directive dans la mesure où il relève de la compétence des Etats membres de définir les détails supplémentaires concernant la durée et les conditions du congé d'aidant. L'interprétation de l'article 188, § 2 du Code du travail indique que tout travailleur bénéficie de deux jours par année civile pour s'absenter du travail, afin d'exercer son droit à un congé d'aidant et porter soin à son enfant. Ceci n'est pas contraire à la directive. Mais l'article 6, al. 2 du texte européen propose un système plus avantageux de congé d'aidant pour le travailleur puisqu'il devient possible d'accorder le congé d'aidant basé sur une période de référence autre qu'une année, par personne ayant besoin de soins ou d'aide, ou par événement. Peut-être serait-il intéressant de transposer ces dispositions dans le Code du travail polonais?

La rémunération que le travailleur devrait percevoir durant son absence du travail est compensée par une allocation, tant en cas de congé de paternité qu'en cas de congé parental et congé d'aidant, ce qui est conforme au contenu de l'article 8 de la directive.

L'article suivant instaure un instrument qui peut s'avérer particulièrement efficace pour maintenir les femmes sur le marché du travail face à l'exercice parallèle du travail d'aidant. La demande formulée par un parent souhaitant bénéficier de formules souples de travail n'est en effet pas connue sous cette forme dans le système polonais. Pourtant, l'article 142 du Code du travail dispose que : "sur demande écrite du travailleur, l'employeur peut définir une formule individuelle de son travail, dans le cadre d'un système de travail concernant ledit travailleur ».

Néanmoins, cette disposition reste trop peu développée pour que l'employeur polonais, examinant une telle demande, lui accorde une attention suffisante et apporte le soutien nécessaire aux personnes assurant les soins à leurs enfants.

La doctrine ajoute même que "cette demande doit être considérée comme une invitation aux négociations dans le cadre desquelles les parties peuvent convenir des conditions et de la forme de cet aménagement spécial du temps de travail ». A cet égard, la directive met en place, dans l'article 9, al. 2, une obligation de tenir compte des besoins des deux parties, et de justifier tout refus d'une telle demande ou de report de ces formules de travail. Il convient de souligner que si jamais les formules souples de travail demandent un effort - tant physique que psychique - trop important face à ses obligations familiales, le travailleur doit toujours avoir la possibilité de renoncer à ces formules de travail. La norme énoncée dans l'article 142 du Code du travail ne permet de mettre en place un aménagement individuel du temps de travail que dans le cadre du système en vigueur : «La loi autorise par exemple de modifier l'heure de début et de fin du travail ou de prévoir des pauses, elle ne permet pas par contre de prolonger la norme maximale du temps de travail quotidien $»^{7}$.

6 K. Stefański, « Art. 142 Indywidualny rozkład czasu pracy » [Formule individuelle du temps de travail], in Kodeks pracy [Code du travail], Komentarze [Commentaires], éd. IV; réd: K.W. Baran; Wolters Kluwer Polska 2018.

7 Ibid. 
En outre, l'article 9, al. 3 de la directive permet au travailleur d'annuler son choix, si la formule souple de travail est d'une durée limitée et lorsqu'un changement de circonstances le justifie. Il est maintenant difficile de dire à quel point cette disposition correspond aux difficultés potentielles présentées ci-dessus. Mais il semble toutefois nécessaire qu'au moment de la mise en œuvre, le législateur polonais prenne en compte cette question.

L'article 10 de la directive régularise les droits en matière d'emploi liés aux congés susmentionnés, tels que la règle de protection des droits acquis par le travailleur, le droit de retrouver son emploi ou un poste de travail équivalent, et le droit de maintenir les prestations de sécurité sociale durant la période de congé. Fort heureusement, les règles régissant en Pologne le congé de paternité, le congé parental et les congés d'aidants ne sont pas contraires à ces droits. La durée du congé annuel n'est pas réduite et le temps de ces congés est pris en compte pour calculer l'ancienneté. Au terme de ces congés, l'employeur doit permettre au travailleur de retrouver le même poste (ou, si cela s'avère impossible, un poste de travail équivalent à celui qu'il occupait avant son départ en congé, ou tout autre poste correspondant à ses qualifications professionnelles, avec une rémunération identique à celle qu'il aurait perçue s'il n'était pas parti en congé) et de voir ses contributions au régime des pensions régulièrement versées.

L'article 12 de la directive devrait élargir ces dispositions en imposant aux Etats membres de prendre les mesures préparatoires nécessaires pour interdire le licenciement des travailleurs au motif qu'ils auraient demandé un congé. Si le travailleur est salarié sur la base d'un contrat à durée indéterminée, il peut se défendre en invoquant l'article $45 \S 1$ du Code du travail, qui impose que toute résiliation du contrat de travail soit justifiée.

Cependant, cette disposition ne s'applique pas aux travailleurs employés sur la base d'un contrat à durée déterminée, ou en période d'essai. Par conséquent, l'employeur n'est pas tenu de justifier sa décision dans la formule de licenciement, bien que le motif réel de licenciement puisse être effectivement lié à la demande du travailleur de pouvoir exercer son droit au congé. Pour améliorer la situation juridique des travailleurs-parents et accorder le droit polonais à la directive, ces dispositions devraient probablement être développées.

L'article 281, point 5 du Code du travail sanctionne l'employeur qui agit contrairement aux dispositions régissant l'octroi de ces congés : "Tout employeur ou toute personne agissant en son nom qui décide, contrairement à la législation relative au temps du travail ou aux droits parentaux des travailleurs et à l'emploi des mineurs, est passible d'une amende allant de 1.000 PLN à 30.000 PLN ». Selon des études menées en 20148, l'un des cas les plus répandus en Pologne de harcèlement des travailleurs par les employeurs réside dans le fait de refuser les demandes de congés. Il est vrai que la Fondation n'a pas opéré de distinction entre les congés pour vacances, et les congés liés à la situation de parents. Néanmoins, un répondant sur dix participant à l'enquête a confirmé avoir été discriminé en raison de son sexe ou de sa situation familiale?. Par conséquent, il ne peut être exclu que les travailleurs-parents soient effectivement concernés par un tel traitement défavorable. II semble donc que les sanctions devraient être plus sévères pour être réellement efficaces, proportionnelles et dissuasives.

8 Etudes présentées par la Fondation Centre de recherche sur l'opinion publique, Communiqué n¹09/2014 intitulé « Harcèlement au travail », 2014.

9 M. Omyłka-Rudzka, «Komunikat z badań CBOS - Szykany w miejscu Pracy » [Communiqué sur les études conduites par le CEOP (CBOS) - Harcèlement au travail], n¹09/2014, Varsovie, 2014. 


\section{MISE EN GUVRE DE LA DIRECTIVE 2019/1158 EN POLOGNE}

La mise en œuvre de l'article 15 de la directive risque de poser un problème car la Pologne ne dispose pas d'organisme de promotion de l'égalité tel que mentionné à l'article 20 de la directive 2006/54/CE ${ }^{10}$. La promotion, l'analyse, le monitoring et le soutien à l'égalité de traitement entre toutes les personnes, sans discrimination fondée sur le sexe, sont actuellement assurés par des organismes ou établissements tels que les inspections du travail, le ministère de la Famille, du Travail et de la Politique sociale, ou encore le Médiateur (considéré par le réseau European Network of Equality Bodies comme un organe correspondant à celui mentionné dans la directive $\left.{ }^{11}\right)$. En tenant compte de la situation légale actuelle, tous les cas de discrimination devront être signalés à l'Inspection nationale du travail ou aux tribunaux de travail.

En conclusion, les études sociologiques confirment qu'il est nécessaire d'engager en Pologne de nouvelles modifications de la loi concernant les aidants et l'égalité des chances femmes-hommes, tant au regard de leur accès au marché du travail qu'en matière d'éducation.

Durant l'épidémie de COVID-19, le législateur a instauré, pour toute cette période de fermeture des écoles, crèches et autres halte-garderie, un dispositif permettant d'obtenir une allocation d'aidant, utilisé par beaucoup de pères. II reste à penser que cette expérience permettra à la société d'avoir un regard plus favorable sur le fait que les hommes puissent être en charge d'obligations parentales de manière égale aux femmes, et que les modifications liées à la mise en œuvre de la directive 2019/1158 soient ainsi jugées positivement.

10 Avis juridique établi par Robert Grzeszczak (Université de Varsovie), pour l'Association Polonaise de la loi sur la lutte contre les discriminations, 6 novembre 2013.

11 Voir https://equineteurope.org/author/poland hrd/

\section{ANNA MUSIALA}

Professeur, Université de Poznań.

Thèmes de recherche: Emploi des jeunes, travail précaire, travail économiquement dépendant.

\section{Publications:}

A. Musiala, " Problèmes juridiques du travail réalisé par un employé exerçant une activité mais dépendant au sens économique », Monitor Prawa Pracy [Moniteur du droit du travail], 2/2014, p. 14.

A. Musiala, «Interprétation des sources autonomes du droit du travail », Przegląd Sądowy [Revue judiciaire], 7-8/2014, p. 80 . 